\title{
Generalized Composition Operators on Zygmund-Orlicz Type Spaces and Bloch-Orlicz Type Spaces
}

\author{
Congli Yang, ${ }^{1}$ Fangwei Chen, ${ }^{2}$ and Pengcheng $\mathrm{Wu}^{1}$ \\ ${ }^{1}$ School of Mathematics and Computer Science, Guizhou Normal University, Guiyang, Guizhou 550001, China \\ ${ }^{2}$ Department of Mathematics and Statistics, Guizhou University of Finance and Economics, Guiyang, Guizhou 550004, China
}

Correspondence should be addressed to Congli Yang; yangcongli@gznu.edu.cn

Received 5 August 2014; Accepted 14 November 2014; Published 30 November 2014

Academic Editor: Naseer Shahzad

Copyright (c) 2014 Congli Yang et al. This is an open access article distributed under the Creative Commons Attribution License, which permits unrestricted use, distribution, and reproduction in any medium, provided the original work is properly cited. \begin{abstract}
The boundedness and compactness of generalized composition operators on Zygmund-Orlicz type spaces and Bloch-Orlicz type
\end{abstract} spaces are established in this paper.

\section{Introduction}

Let $\mathbb{D}$ be a unit disk in complex plane $\mathbb{C}$, and let $H(\mathbb{D})$ be the space of all holomorphic functions on $\mathbb{D}$ with the topology of uniform convergence on compact subsets of $\mathbb{D}$. The Bloch space, $\mathscr{B}$, consists of all functions $f \in H(\mathbb{D})$ for which

$$
\|f\|_{b}:=\sup _{z \in \mathbb{D}}\left(1-|z|^{2}\right)\left|f^{\prime}(z)\right|<\infty
$$

$\mathscr{B}$ becomes a Banach space when it is equipped with the norm $\|f\|_{\mathscr{B}}:=|f(0)|+\|f\|_{b}$ (see, e.g., [1]).

Let $\alpha>0$, and the $\alpha$-Bloch space, denoted by $\mathscr{B}^{\alpha}$, consists of all holomorphic functions $f$ on $\mathbb{D}$ such that

$$
\|f\|_{\alpha}:=\sup _{z \in \mathbb{D}}\left(1-|z|^{2}\right)^{\alpha}\left|f^{\prime}(z)\right|<\infty .
$$

$\alpha$-Bloch space is introduced and studied by numerous authors. The general theory of $\alpha$-Bloch function spaces is referred to in [2]. Recently, many authors studied different class of Bloch-type spaces, where the typical weight function, $w(z)=1-|z|^{2}(z \in \mathbb{D})$, is replaced by a bounded continuous positive function $\mu$ defined on $\mathbb{D}$. More precisely, a function $f \in H(\mathbb{D})$ is called a $\mu$-Bloch function, denoted by $f \in \mathscr{B}^{\mu}$, if

$$
\|f\|_{\mu}:=\sup _{z \in \mathbb{D}} \mu(z)\left|f^{\prime}(z)\right|<\infty .
$$

Clearly, if $\mu(z)=w(z)^{\alpha}$ with $\alpha>0, \mathscr{B}^{\mu}$ is just the $\alpha$-Bloch space $\mathscr{B}^{\alpha}$. It is readily seen that $\mathscr{B}^{\mu}$ is a Banach space with the norm

$$
\|f\|_{\mathscr{B}^{\mu}}:=|f(0)|+\|f\|_{\mu} .
$$

$\mathscr{B}^{\mu}$ spaces appear in the literature of a natural way when one studies the properties of some operators of holomorphic functions in a certain space. For instance, Attele in [3] proves that the Hankel operator induced by a function $f$ in the Bergman space is bounded if and only if $f \in \mathscr{B}^{\mu_{1}}$ (where $\mu_{1}=$ $w(z) \log (z / w(z))$, with $z \in \mathbb{D})$. The space $\mathscr{B}^{\mu_{1}}$ is known as the log-Bloch space or the weighted Bloch space. The logarithmic Bloch-type space is introduced by Krantz and Stević [4, 5], where some properties of this space are studied. In the last decade, there was a big interest in the investigation of Blochtype spaces and various concrete linear operators $L: X \rightarrow Y$, where at least one of the spaces $X$ and $Y$ is Bloch space. For some other recent results in the areas, see, for example, $[4,6-$ $10]$ and a lot of references therein.

Recently, Fernándz in [8] uses Young's functions to define the Bloch-Orlicz space as a generalization of Bloch space. More precisely, let $\varphi:[0, \infty) \rightarrow[0, \infty)$ be an $\mathcal{N}$-function; that is, $\varphi$ is a strictly increasing convex function such that $\varphi(0)=0$ and $\lim _{t \rightarrow \infty}(t / \varphi(t))=\lim _{t \rightarrow 0}(\varphi(t) / t)=0$. The Bloch-Orlicz space associated with the function $\varphi$, denoted by $\mathscr{B}^{\varphi}$, is the class of all analytic functions $f \in \mathbb{D}$ such that

$$
\sup _{z \in \mathbb{D}}\left(1-|z|^{2}\right) \varphi\left(\lambda\left|f^{\prime}(z)\right|\right)<\infty,
$$


for some $\lambda>0$ depending on $f$. Without loss of generality, we can suppose that $\varphi^{-1}$ is continuous and differentiable. In fact, if $\varphi^{-1}$ is not differentiable everywhere, we set the function

$$
\psi(t)=\int_{0}^{t} \frac{\varphi(x)}{x} d x \quad(t \geq 0)
$$

then $\varphi$ is differentiable, whence $\psi^{-1}$ is differentiable everywhere on $[0, \infty)$. Furthermore, $\varphi$ is a strictly increasing and convex function satisfying $\varphi(0)=0$; then the function $\varphi(t) / t$, $t>0$, is increasing and

$$
\varphi(t) \geq \psi(t) \geq \int_{t / 2}^{t} \frac{\varphi(x)}{x} d x \geq \varphi\left(\frac{t}{2}\right)
$$

for all $t \geq 0$. Hence $\mathscr{B}^{\varphi}=\mathscr{B}^{\psi}$. By the convexity of $\varphi$, it is not difficult to see that the Minkowski's functional

$$
\|f\|_{b^{\varphi}}=\inf \left\{k>0: S_{\varphi}\left(\frac{f^{\prime}}{k}\right) \leq 1\right\}
$$

defines a seminorm for $\mathscr{B}^{\varphi}$, which in this case is known as Luxemburg's seminorm, where

$$
S_{\varphi}(f):=\sup _{z \in \mathbb{D}}\left(1-|z|^{2}\right) \varphi(|f(z)|) .
$$

In fact, it can be shown that $\mathscr{B}^{\varphi}$ is a Banach space with the norm

$$
\|f\|_{\mathscr{B}^{\varphi}}=|f(0)|+\|f\|_{b^{\varphi}} .
$$

We observe that, for any $f \in \mathscr{B}^{\varphi} \backslash\{0\}$, the following relation

$$
S_{\varphi}\left(\frac{f^{\prime}}{\|f\|_{\mathscr{B}^{\varphi}}}\right) \leq 1
$$

holds.

The inequality above allows us to obtain

$$
\left|f^{\prime}(z)\right| \leq \varphi^{-1}\left(\frac{1}{1-|z|^{2}}\right)\|f\|_{\mathscr{B}^{\varphi}},
$$

for all $f \in \mathscr{B}^{\varphi}$ and for all $z \in \mathbb{D}$. This last inequality implies that the evaluation function is defined as $T_{z}(f):=f(z)$, where $z \in \mathbb{D}$ is fixed and $f \in \mathscr{B}^{\varphi}$ is continuous on $\mathscr{B}^{\varphi}$. In fact, let $z \in \mathbb{D}$ be fixed and any $f \in \mathscr{B}^{\varphi}$; we have

$$
\begin{aligned}
\left|T_{z}(f)\right| & =|f(z)| \leq|f(0)|+\int_{[0, z]}\left|f^{\prime}(s)\right||d s| \\
& \leq\left(1+\int_{0}^{1} \varphi^{-1}\left(\frac{1}{1-|z|^{2} t^{2}}\right) d z\right)\|f\|_{\mathscr{B}^{\varphi}} .
\end{aligned}
$$

From the definition of Luxemburg seminorm and the expression (11), we have

$$
S_{\varphi}\left(f^{\prime}\right) \leq 1 \Longleftrightarrow\|f\|_{\mathscr{B}^{\varphi}} \leq 1
$$

for any $f \in \mathscr{B}^{\varphi}$.
As an easy consequence of (11), we have that the BlochOrlicz space is isometrically equal to $\mu$-Bloch space when

$$
\mu(z)=\frac{1}{\varphi^{-1}\left(1 /\left(1-|z|^{2}\right)\right)},
$$

with $z \in \mathbb{D}$. Thus, for any $f \in \mathscr{B}^{\varphi}$, we have

$$
\|f\|_{\mathscr{B}^{\varphi}}=\sup _{z \in \mathbb{D}} \mu(z)\left|f^{\prime}(z)\right| .
$$

Denote by $\mathscr{Z}$ the class of all $f \in H(\mathbb{D}) \cap C(\overline{\mathbb{D}})$ such that

$$
\|f\|=\sup \frac{\left|f\left(e^{i(\theta+h)}\right)+f\left(e^{i(\theta-h)}\right)-2 f\left(e^{i \theta}\right)\right|}{h}<\infty \text {, }
$$

where the supremum is taken over all $e^{i \theta} \in \partial \mathbb{D}$ and $h>0$. From the theorem of Zygmund (see [11, Theroem 5.3]) and the closed graph theorem, we see that $f \in \mathscr{Z}$ if and only if $\sup _{z \in \mathbb{D}}\left(1-|z|^{2}\right)\left|f^{\prime \prime}(z)\right|<\infty$. It is easy to see that $\mathscr{Z}$ is a Banach space under the norm $\|\cdot\|_{\mathscr{E}}$, where

$$
\|f\|_{\mathscr{X}}=|f(0)|+\left|f^{\prime}(0)\right|+\sup _{z \in \mathbb{D}}\left(1-|z|^{2}\right)\left|f^{\prime \prime}(z)\right| .
$$

From (18) it is easy to obtain

$$
\left|f^{\prime}(z)-f^{\prime}(0)\right| \leq \frac{1}{2}\|f\|_{\mathscr{Z}} \log \frac{1+|z|}{1-|z|} \quad \text { for } f \in \mathscr{Z} .
$$

For some other information and operators on this space, see, for example, $[6,12,13]$.

Inspired by the way Bloch-Orlicz spaces were defined (see $[8,14]$ ), we define the Zygmund-Orlicz space, which is denoted by $\mathscr{Z}^{\varphi}$, as the class of all analytic functions $f$ in $\mathbb{D}$ such that

$$
\sup _{z \in \mathbb{D}}\left(1-|z|^{2}\right) \varphi\left(\lambda\left|f^{\prime \prime}(z)\right|\right) \leq \infty
$$

for some $\lambda>0$ depending on $f$. Same as the Bloch-Orlicz space, since $\varphi$ is convex, it is not difficult to see that the Minkowski functional

$$
\|f\|_{z^{\varphi}}=\inf \left\{k>0: S_{\varphi}\left(\frac{f^{\prime \prime}}{k}\right) \leq 1\right\}
$$

defines a seminorm for $\mathscr{Z}^{\varphi}$. Furthermore, it can be shown that $\mathscr{Z}^{\varphi}$ is a Banach space with the norm

$$
\|f\|_{\mathscr{Z}^{\varphi}}=|f(0)|+\left|f^{\prime}(0)\right|+\|f\|_{z^{\varphi}} .
$$

The following useful lemmas are easily obtained.

Lemma 1 (see [15]). If $f \in \mathscr{Z}$, then

(i) $|f(z)| \leq\|f\|_{\mathscr{E}} \quad$ for every $z \in \mathbb{D}$,

(ii) $\left|f^{\prime}(z)\right| \leq \log \left(\frac{e}{1-|z|^{2}}\right)\|f\|_{\mathscr{Z}} \quad$ for every $z \in \mathbb{D}$. 
Also we can observe the following property.

Lemma 2. For any $f \in \mathscr{Z}^{\varphi} \backslash\{0\}$, the following relation

$$
S_{\varphi}\left(\frac{f^{\prime \prime}}{\|f\|_{\mathscr{Z}^{\varphi}}}\right) \leq 1
$$

holds.

Proof. In the same way as the case of Bloch-Orlicz space, so the details are omitted here.

Lemma 2 allows us to obtain that

$$
\left|f^{\prime \prime}(z)\right| \leq \varphi^{-1}\left(\frac{1}{1-|z|^{2}}\right)\|f\|_{\mathscr{E}^{\varphi}},
$$

for all $f \in \mathscr{Z}^{\varphi}$ and all $z \in \mathbb{D}$.

From the definition of Luxemburg seminorm and expression (25), we have

$$
S_{\varphi}\left(f^{\prime \prime}\right) \leq 1 \Longleftrightarrow\|f\|_{\mathscr{Z}^{\varphi}} \leq 1
$$

Also, as an easy consequence of (25), we have that ZygmundOrlicz space is isometrically equal to $\mu$-Zygmund space, where $\mu(z)=1 / \varphi^{-1}\left(1 /\left(1-|z|^{2}\right)\right)$ with $z \in \mathbb{D}$. For more information about $\mathscr{Z}^{\mu}$, see $[7,10,16]$.

Specially, if $\varphi$ is an $\mathcal{N}$-function such that

$$
I_{\varphi}(z)=\int_{0}^{1} \varphi^{-1}\left(\frac{1}{1-|z|^{2} t^{2}}\right) d t
$$

is bounded for all $z \in \mathbb{D}$, then we get $\mathscr{Z}^{\varphi} \subset H^{\infty}$, the space of all bounded analytic functions on $\mathbb{D}$. However, there exists $\mathcal{N}$-functions for which $I_{\varphi}(z)$ is not a bounded function; for instance, consider $\varphi(t)=t \log (1+t)$ with $t \geq 0$.

Let $\phi$ be an analytic self-map of $\mathbb{D}$; then the composition operator on $H(\mathbb{D})$ is given by

$$
C_{\phi} f=f \circ \phi
$$

Composition operators acting on various spaces of analytic functions have been the object for recent years. In particular, the problems of relating operator-theoretic properties of $C_{\phi}$ to function-theoretic properties of $\phi$ are interesting and have been widely discussed. See the book of Cowen and MacCluer [17] and Shapiro [9] for discussions of composition operators classical spaces of analytic functions.

Assume that $g: \mathbb{D} \rightarrow \mathbb{C}$ is a holomorphic map of the disk $\mathbb{D}$, for $f \in H(\mathbb{D})$; we define a linear operator as follows:

$$
\left(C_{\phi}^{g} f\right)(z)=\int_{0}^{z} f^{\prime}(\phi(\xi)) g(\xi) d \xi, \quad z \in \mathbb{D} .
$$

The operator $C_{\phi}^{g}$ is called the generalized composition operator, when $g=\phi^{\prime}$. We see that this operator is essentially composition operator, since the difference $C_{\phi}^{g}-C_{\phi}$ is constant. Therefore, $C_{\phi}^{g}$ is a generalization of the composition operator, which was introduced in [6].
Recall that if $X$ and $Y$ are Banach spaces and $L: X \rightarrow$ $Y$ is a linear operator, then $L$ is said to be compact if, for every bounded sequence $\left\{x_{n}\right\}$ in $X$, the sequence $\left(L\left\{x_{n}\right\}\right)$ has a convergent subsequence. The book [17] contains plenty of information on this topic. By the standard arguments (see, e.g., Proposition 3.11 in [17]), the following lemma follows.

Lemma 3. Let $\varphi:[0, \infty) \rightarrow[0, \infty)$ be an $\mathcal{N}$-function, $g \in$ $H(\mathbb{D})$, and $\phi$ an analytic self-map of $\mathbb{D}$. Let $X=\mathscr{Z}$ and $Y=$ $\mathscr{B}^{\varphi}$ or $\mathscr{Z}^{\varphi}$. Then $C_{\phi}^{g}: X \rightarrow Y$ is compact if and only if $C_{\phi}^{g}$ : $X \rightarrow Y$ is bounded and, for any bounded sequence $\left(f_{k}\right)_{k \in N}$ in $X$ which converges to zero uniformly on $\mathbb{D}$ as $k \rightarrow \infty$, one has $\left\|C_{\phi}^{g} f_{k}\right\|_{Y} \rightarrow 0$ as $k \rightarrow \infty$.

Some characterization of the boundedness and compactness of the composition operator, as well as Volterra type operator, on Bloch-Orlicz-type space and Zygmund space can be found in [2,18-22]. In [6], the boundedness and compactness of the generalized composition operator on Zygmund space and Bloch-type spaces are characterized by Li and Stević.

In this paper, we are devoted to investigating the boundedness and compactness of generalized composition operators between Zygmound-Orlicz type spaces and BlochOrlicz type spaces. The paper is organized as follows. In Section 2 we give the necessary and sufficient conditions for the boundedness and compactness of the operator $C_{\phi}^{g}$ : $\mathscr{Z} \rightarrow \mathscr{B}^{\varphi}$. In Section 3 we obtain the necessary and sufficient conditions for the boundedness and compactness of the operator $C_{\phi}^{g}$ on Zygmund type spaces. Throughout this paper, we use the letter $C$ to denote a generic positive constant that can change its value at each occurrence. The notation $a \leq b$ means that there is a positive constant $C$ such that $a \leq C b$. If $a \leq b$ and $b \leq a$ hold, then one says that $a \asymp b$.

\section{The Boundedness and Compactness of $C_{\phi}^{g}: \mathscr{Z} \rightarrow \mathscr{B}^{\varphi}$}

Now, we are ready to state and prove the main results in this section.

Theorem 4. Let $\varphi:[0, \infty) \rightarrow[0, \infty)$ be an $\mathcal{N}$-function, $g \in$ $H(\mathbb{D})$, and $\phi$ an analytic self-map of $\mathbb{D}$. Then $C_{\phi}^{g}: \mathscr{Z} \rightarrow \mathscr{B}^{\varphi}$ is bounded if and only if

$$
\begin{aligned}
L & :=\sup _{z \in \mathbb{D}}\left(\varphi^{-1}\left(\frac{1}{1-|z|^{2}}\right)\right)^{-1}|g(z)| \log \left(\frac{e}{1-|\phi(z)|^{2}}\right) . \\
& <\infty .
\end{aligned}
$$

Proof. Suppose that (31) holds. For arbitrary $z \in \mathbb{D}$ and $f \in$ $\mathscr{Z}$, by Lemma 1 , we have the following estimate:

$$
S_{\varphi}\left(\frac{\left(C_{\phi}^{g} f\right)^{\prime}(z)}{L\|f\|_{\mathscr{Z}}}\right)
$$




$$
\begin{aligned}
= & \sup _{z \in \mathbb{D}}\left(1-|z|^{2}\right) \varphi\left(\frac{\left|f^{\prime}(\phi(z))\right||g(z)|}{L\|f\|_{\mathscr{Z}}}\right) \\
\leq & \sup _{z \in \mathbb{D}}\left(1-|z|^{2}\right) \varphi \\
& \times\left(\varphi^{-1}\left(\frac{1}{1-|z|^{2}}\right) \frac{\left|f^{\prime}(\phi(z))\right|}{\log \left(e /\left(1-|\phi(z)|^{2}\right)\right)\|f\|_{\mathscr{Z}}}\right) \\
\leq & \sup _{z \in \mathbb{D}}\left(1-|z|^{2}\right) \varphi\left(\varphi^{-1}\left(\frac{1}{1-|z|^{2}}\right)\right) \leq 1,
\end{aligned}
$$

where we use Lemma 1 (relation (ii)) in the last inequality. From here, we can conclude that $\left\|C_{\phi}^{g}\right\|_{\varphi} \leq L\|f\|_{\mathscr{X}}$. Since $C_{\phi}^{g} f(0)=0$, it follows that the generalized composition operator $C_{\phi}^{g}: \mathscr{Z} \rightarrow \mathscr{B}^{\varphi}$ is bounded.

Now, suppose that there exists a constant $C>0$ such that

$$
\left\|C_{\phi}^{g}\right\| \varphi \leq C\|f\|_{\mathscr{Z}} \quad \forall f \in \mathscr{Z} .
$$

Let

$$
h(z)=(z-1)\left[\left(1+\log \frac{e}{1-z}\right)^{2}+1\right]
$$

and put

$$
f_{a}(z)=\frac{h(\bar{a} z)}{\bar{a}}\left(\log \frac{e}{1-|a|^{2}}\right)^{-1},
$$

for any $a \in \mathbb{D}$ such that $1 / \sqrt{2}<|a|<1$. Then we have

$$
\begin{aligned}
& f_{a}^{\prime}(z)=\left(\log \frac{e}{1-\bar{a} z}\right)^{2}\left(\log \frac{e}{1-|a|^{2}}\right)^{-1}, \\
& f_{a}^{\prime \prime}(z)=\frac{2 \bar{a}}{1-\bar{a} z}\left(\log \frac{e}{1-\bar{a} z}\right)\left(\log \frac{e}{1-|a|^{2}}\right)^{-1},
\end{aligned}
$$

which implies that

$$
\begin{aligned}
\left|f_{a}^{\prime \prime}(z)\right| & \leq \frac{2}{1-|z|}\left(C+\log \frac{e}{1-|a|}\right)\left(\log \frac{e}{1-|a|^{2}}\right)^{-1} \\
& \leq \frac{2}{1-|z|},
\end{aligned}
$$

for $1 / \sqrt{2}<|a|<1$ and $\sup _{1 / \sqrt{2}<|a|<1}\left\|f_{a}\right\|_{\mathscr{Z}}<\infty$. Therefore we have

$$
\left\|C_{\phi}^{g} f_{a}\right\|_{\varphi} \leq C\left\|f_{a}\right\|_{\mathscr{Z}} \leq L<\infty .
$$

It follows that

$$
S_{\varphi}\left(\frac{\left(C_{\varphi}^{g} f_{a}\right)^{\prime}(z)}{L}\right) \leq 1 .
$$

That is,

$$
\left(1-|z|^{2}\right) \varphi\left(\frac{\left(C_{\varphi}^{g} f_{a}\right)^{\prime}(z)}{L}\right) \leq 1,
$$

for any $a \in \mathbb{D}$. But, on the other hand,

$$
\varphi\left(\frac{|g(z)|}{L}\left|f_{a}^{\prime}(\phi(z))\right|\right) \leq \frac{1}{1-|z|^{2}}
$$

and then

$$
\frac{|g(z)|}{L}\left|f_{a}^{\prime}(\phi(z))\right| \leq \varphi^{-1}\left(\frac{1}{1-|z|^{2}}\right)
$$

So we have

$$
\left(\varphi^{-1}\left(\frac{1}{1-|z|^{2}}\right)\right)^{-1}|g(z)|\left|f_{a}^{\prime}(\phi(z))\right| \leq L,
$$

for all $a \in \mathbb{D}$. In particular, for $a=\phi(z)$, we have

$$
\left(\varphi^{-1}\left(\frac{1}{1-|z|^{2}}\right)\right)^{-1}|g(z)| \log \frac{e}{1-|\phi(z)|^{2}} \leq L .
$$

This concludes the proof of the theorem.

Theorem 5. Let $\varphi:[0, \infty) \rightarrow[0, \infty)$ be an $\mathcal{N}$-function, $g \in$ $H(\mathbb{D})$, and $\phi$ an analytic self-map of $\mathbb{D}$. Then $C_{\phi}^{g}: \mathscr{Z} \rightarrow \mathscr{B}^{\varphi}$ is compact if and only if $C_{\phi}^{g}: \mathscr{Z} \rightarrow \mathscr{B}^{\varphi}$ is bounded and

$$
\lim _{|\phi(z)| \rightarrow 1} \mu(z)|g(z)| \log \left(\frac{e}{1-|\phi(z)|^{2}}\right)=0 .
$$

Proof. First assume that $C_{\phi}^{g}: \mathscr{Z} \rightarrow \mathscr{B}^{\varphi}$ is bounded and (45) holds. By the boundedness of $C_{\varphi}^{g}$ with $f(z)=z$, we see that

$$
L:=\sup _{z \in \mathbb{D}} \mu(z)|g(z)|<\infty .
$$

Let $\left\{f_{n}\right\}$ be a sequence in $\mathscr{Z}$ such that $\sup _{n \in N}\left\|f_{n}\right\|_{\mathscr{Z}} \leq K$ and $f_{n} \rightarrow 0$ uniformly on compact subsets of $\mathbb{D}$ as $n \rightarrow \infty$. Then, by Lemma 3, it suffices to show that $\left\|C_{\phi}^{g} f_{n}\right\|_{\mu} \rightarrow 0$ as $n \rightarrow \infty$. By (45), we have that, for every $\varepsilon>0$, there is a constant $r \in(0,1)$, such that $r<|\phi(z)| \leq 1$, which implies

$$
\mu(z)|g(z)| \log \left(\frac{e}{1-|\phi(z)|^{2}}\right)<\frac{\varepsilon}{k},
$$

for any $z \in \mathbb{D}$. From here, we have that

$$
\begin{aligned}
& \mu(z)\left|\left(C_{\phi}^{g} f_{n}\right)^{\prime}(z)\right| \\
& \quad=\mu|g(z)|\left|f_{n}^{\prime}(\phi(z))\right| \\
& \quad \leq \mu(z)|g(z)| \log \left(\frac{e}{1-|\phi(z)|^{2}}\right)\left\|f_{n}\right\|_{\mathscr{Z}} \leq \varepsilon,
\end{aligned}
$$

where $r<|\phi(z)|<1$.

On the other hand, let $\Omega=\{\omega \in \mathbb{D}:|\omega| \leq r\}$; by the cauchy estimate, if $\left\{f_{n}\right\}_{n \in N}$ is a sequence converging to zero on compact subsets of $\mathbb{D}$, then the sequence $\left\{f_{n}^{\prime}\right\}_{n \in N}$ also converges to zero on compact subsets of $\mathbb{D}$ as $n \rightarrow \infty$. In particular, since $\Omega$ is compact, it follows that $\lim _{n \rightarrow \infty} \sup _{\omega \in \Omega}\left|f_{n}^{\prime}(\omega)\right|=0$. Using these facts and letting $n \rightarrow \infty$, we obtain

$$
\begin{aligned}
& \sup _{|\phi(z)| \leq r} \mu(z)\left|\left(C_{\phi}^{g} f_{n}\right)^{\prime}(z)\right| \\
& \quad=\sup _{|\phi(z)| \leq r} \mu(z)|g(z)|\left|f_{n}^{\prime}(\phi(z))\right| \\
& \quad \leq L \sup _{\omega \in \Omega}\left|f_{n}^{\prime}(\omega)\right| \longrightarrow 0, \quad \text { as } n \longrightarrow \infty .
\end{aligned}
$$


Since $\left(C_{\phi}^{g} f_{n}\right)(0) \rightarrow 0$ as $n \rightarrow \infty$, for given $\varepsilon>0$, there exists an $n \in N$, such that

$$
\left\|C_{\phi}^{g} f_{n}\right\|_{\mu}=\left|C_{\phi}^{g} f_{n}(0)\right|+\sup _{z \in \mathbb{D}} \mu(z)\left|\left(C_{\phi}^{g} f_{n}\right)^{\prime}(z)\right| \leq 3 \varepsilon,
$$

whenever $n \geq N$, which means that $C_{\phi}^{g}: \mathscr{Z} \rightarrow \mathscr{B}^{\varphi}$ is a compact operator.

To prove the converse, suppose that there exists an $\varepsilon_{0}>0$ such that

$$
\sup _{|\phi(z)| \geq r} \mu(z)|g(z)| \log \left(\frac{e}{1-|\phi(z)|^{2}}\right) \geq \varepsilon_{0},
$$

for any $r \in(0,1)$. Given a sequence of real number $\left\{r_{n}\right\} \subset$ $(0,1)$ such that $r_{n} \rightarrow 1$ as $n \rightarrow \infty$, we can find a sequence $\left\{z_{n}\right\} \subset \mathbb{D}$ such that $\left|\phi\left(z_{n}\right)\right| \geq r_{n}$ and

$$
\mu\left(z_{n}\right)\left|g\left(z_{n}\right)\right| \log \left(\frac{e}{1-|\omega|^{2}}\right) \geq \frac{1}{2} \varepsilon_{0},
$$

where $\omega_{n}=\phi\left(z_{n}\right)$, and, if necessary, we may suppose that $\left|\omega_{n}\right| \rightarrow 1$ as $n \rightarrow \infty$.

Note that $f_{a}$, defined by (35), converges to zero uniformly on compact subset of $\mathbb{D}$ as $|a| \rightarrow 1$ and

$$
f_{a}^{\prime}(a)=\log \frac{e}{1-|a|^{2}}, \quad \text { for } a \in \mathbb{D} \backslash\{0\} .
$$

Now, we choose functions $\left\{f_{n}\right\}_{n \in N}$ defined by

$$
\begin{aligned}
& f_{n}(z) \\
& =\frac{\bar{w}_{n} z-1}{\bar{w}}\left[\left(1+\log \frac{e}{1-\overline{w_{n}} z}\right)^{2}+1\right]\left[\log \frac{e}{1-\left|w_{n}\right|^{2}}\right]^{-1} .
\end{aligned}
$$

From the proof of Theorem 4, we see that

$$
\sup _{n \in N}\|f\|_{\mathscr{Z}} \leq C .
$$

Moreover, we can see that $\left\{f_{n}\right\}$ converges to zero uniformly on compact subsets of $\mathbb{D}$ and satisfies

$$
\begin{aligned}
\left\|C_{\phi}^{g} f_{n}\right\|_{\mu} & \geq \mu\left(z_{n}\right)\left|g\left(z_{n}\right)\right|\left|f_{n}^{\prime}\left(w_{n}\right)\right| \\
& =\mu\left(z_{n}\right)\left|g\left(z_{n}\right)\right| \log \left(\frac{e}{1-\left|w_{n}\right|^{2}}\right) \geq \frac{1}{2} \varepsilon_{0}>0 .
\end{aligned}
$$

Therefore, $C_{\phi}^{g}: \mathscr{Z} \rightarrow \mathscr{B}^{\varphi}$ is not a compact operator. This completes the proof of the theorem.

\section{The Boundedness and Compactness of $C_{\phi}^{g}: \mathscr{Z} \rightarrow \mathscr{Z}^{\varphi}$}

In this section, we characterize the boundedness and compactness of the operator $C_{\phi}^{g}$ on Zygmund-Orlicz type spaces.
Theorem 6. Let $\varphi:[0, \infty) \rightarrow[0, \infty)$ be an $\mathcal{N}$-function, $g \in$ $H(\mathbb{D})$, and $\phi$ an analytic self-map of $\mathbb{D}$. Then $C_{\phi}^{g}: \mathscr{Z} \rightarrow \mathscr{Z}^{\varphi}$ is bounded if and only if

$$
\begin{aligned}
& k_{1}=\sup _{z \in \mathbb{D}} \frac{\left|\phi^{\prime}(z)\right||g(z)|}{\varphi^{-1}\left(1 /\left(1-|z|^{2}\right)\right)\left(1-|\phi(z)|^{2}\right)}<\infty, \\
& k_{2}=\sup _{z \in \mathbb{D}} \frac{\left|g^{\prime}(z)\right| \log \left(e /\left(1-|\phi(z)|^{2}\right)\right)}{\varphi^{-1}\left(1 /\left(1-|z|^{2}\right)\right)\left(1-|\phi(z)|^{2}\right)}<\infty .
\end{aligned}
$$

Proof. Suppose first that (57) holds. For each $f \in \mathscr{Z}$, we have the following estimate:

$$
\begin{aligned}
& S_{\varphi}\left(\frac{\left(C_{\phi}^{g} f\right)^{\prime \prime}(z)}{\|f\|_{\mathscr{I}}}\right) \\
& \leq \sup _{z \in \mathbb{D}}\left(1-|z|^{2}\right) \varphi \\
& \times\left(\frac{\left|f^{\prime \prime}(\phi(z))\right||g(z)|+\left|f^{\prime}(\phi(z))\right|\left|g^{\prime}(z)\right|}{C\|f\|_{\mathscr{X}}}\right) \\
& =\sup _{z \in \mathbb{D}}\left(1-|z|^{2}\right) \varphi \\
& \times\left[\left(\frac{\varphi^{-1}\left(1 /\left(1-|\phi(z)|^{2}\right)\right)\left|f^{\prime \prime}(\phi(z))\right|\left|\phi^{\prime}(z)\right||g(z)|}{\varphi^{-1}\left(1 /\left(1-|z|^{2}\right)\right) C\|f\|_{\mathscr{Z}}}\right)\right. \\
& \left.+\left(\frac{\varphi^{-1}\left(1 /\left(1-|\phi(z)|^{2}\right)\right)\left|f^{\prime}(\phi(z))\right|\left|g^{\prime}(z)\right|}{\varphi^{-1}\left(1 /\left(1-|z|^{2}\right)\right) C\|f\|_{\mathscr{L}}}\right)\right] \\
& \leq \sup _{z \in \mathbb{D}}\left(1-|z|^{2}\right) \varphi \\
& \times\left(\frac{k_{1} \varphi^{-1}\left(1 /\left(1-|z|^{2}\right)\right)\left(1-|\phi(z)|^{2}\right)\left|f^{\prime \prime}(\phi(z))\right|}{C\|f\| \mathscr{Z}}\right. \\
& \left.+\frac{k_{2} \varphi^{-1}\left(1 /\left(1-|z|^{2}\right)\right)\left|f^{\prime}(\phi(z))\right|}{\log \left(e /\left(1-|\phi(z)|^{2}\right)\right) C\|f\|_{\mathscr{X}}}\right) \\
& =\sup _{z \in \mathbb{D}}\left(1-|z|^{2}\right) \varphi\left(\frac{k_{1} C_{1}+k_{2} C_{2}}{C} \varphi^{-1}\left(\frac{1}{1-|z|^{2}}\right)\right) \\
& \leq \sup _{z \in \mathbb{D}}\left(1-|z|^{2}\right) \varphi\left(\varphi^{-1}\left(\frac{1}{1-|z|^{2}}\right)\right)=1,
\end{aligned}
$$

where $C_{1}$ and $C_{2}$ are constants, such that $k_{1} C_{1}+k_{2} C_{2} \leq$ $C$. Here we use relations (26) and (24) in the last seconded inequality. Now, we can conclude that

$$
\left\|C_{\phi}^{g} f\right\|_{\mathscr{E}^{\phi}} \leq C\|f\|_{\mathscr{X}},
$$

and the generalized composition operator $C_{\phi}^{g}: \mathscr{Z} \rightarrow \mathscr{E}$ is bounded. 
Now assume that $C_{\phi}^{g}: \mathscr{Z} \rightarrow \mathscr{Z}$ is bounded; that is, there exists a constant $C$ such that $\left\|C_{\phi}^{g} f\right\|_{\mathscr{X}^{\phi}} \leq C\|f\|_{\mathscr{X}}$ for all $f \in \mathscr{Z}$. Taking the functions $f(z)=z$ and $f(z)=z^{2}$, respectively, we obtain

$$
\left(1-|z|^{2}\right) \varphi\left(\frac{\left|g^{\prime}(z)\right|}{C}\right) \leq 1,
$$

which implies

$$
\begin{gathered}
\sup _{z \in \mathbb{D}} \frac{\left|g^{\prime}(z)\right|}{\varphi^{-1}\left(1 /\left(1-|z|^{2}\right)\right)}<\infty, \\
\left(1-\left(|z|^{2}\right)\right) \varphi\left(\frac{\left|\phi^{\prime}(z)\right||g(z)|+|\phi(z)|\left|g^{\prime}(z)\right|}{C\left(1-|z|^{2}\right)}\right) \leq 1 .
\end{gathered}
$$

Then

$$
\frac{\left|\phi^{\prime}(z)\right||g(z)|+|\phi(z)|\left|g^{\prime}(z)\right|}{\varphi^{-1}\left(1 /\left(1-|z|^{2}\right)\right)} \leq C .
$$

So we obtain

$$
\sup _{z \in \mathbb{D}} \frac{\left|\phi^{\prime}(z)\right||g(z)|+|\phi(z)|\left|g^{\prime}(z)\right|}{\varphi^{-1}\left(1 /\left(1-|z|^{2}\right)\right)} \leq \infty .
$$

Using these facts and the boundedness of the function $\phi(z)$ we get

$$
\sup _{z \in \mathbb{D}} \frac{\left|\phi^{\prime}(z)\right||g(z)|}{\varphi^{-1}\left(1 /\left(1-|z|^{2}\right)\right)}<\infty
$$

Let $h(z)=(z-1)\left[(1+\log (e /(1-z)))^{2}+1\right]$ and put $f_{a}(z)=$ $(h(\bar{a} z) / \bar{a})\left(\log \left(e /\left(1-|a|^{2}\right)\right)\right)^{-1}$ for $a \in \mathbb{D}$ such that $|a|>1 / 2$. We have

$$
\begin{aligned}
\left(1-|\lambda|^{2}\right) \varphi( & \left(\left|g^{\prime}(\lambda)\right| \log \frac{e}{1-|\phi(z)|^{2}}\right. \\
& \left.-2\left|\phi^{\prime}(\lambda)\right||g(\lambda)| \frac{|\phi(\lambda)|}{1-|\phi(z)|^{2}}\right) \\
& \left.\times\left(C|| C_{\phi}^{g} \|_{\mathscr{Z} \rightarrow \mathscr{E}^{\varphi}}\right)^{-1}\right) \leq 1 .
\end{aligned}
$$

\section{Then}

$$
\begin{aligned}
& \left(\left(\left|g^{\prime}(\lambda)\right| \log \frac{e}{1-|\phi(z)|^{2}}-2\left|\phi^{\prime}(\lambda)\right||g(\lambda)| \frac{|\phi(\lambda)|}{1-|\phi(z)|^{2}}\right)\right. \\
& \left.\quad \times\left(C\left\|C_{\phi}^{g}\right\|_{\mathscr{Z} \rightarrow \mathscr{E}^{\varphi}}\right)^{-1}\right) \\
& \quad \leq \varphi^{-1}\left(\frac{1}{1-|\lambda|^{2}}\right), \\
& \frac{\left|g^{\prime}(\lambda)\right|\left|\log \left(e /\left(1-|\phi(\lambda)|^{2}\right)\right)\right|}{\varphi^{-1}\left(1 /\left(1-|\lambda|^{2}\right)\right)} \\
& -2 \frac{\left|\phi^{\prime}(\lambda)\right||g(\lambda)||\phi(\lambda)|}{\varphi^{-1}\left(1 /\left(1-|\lambda|^{2}\right)\right)\left(1-|\phi(\lambda)|^{2}\right)} \leq C\left\|C_{\phi}^{g}\right\|_{\mathscr{X} \rightarrow \mathscr{X}^{\varphi}} .
\end{aligned}
$$

So we obtain

$$
\begin{aligned}
& \frac{\left|g^{\prime}(\lambda)\right|\left|\log \left(e /\left(1-|\phi(\lambda)|^{2}\right)\right)\right|}{\varphi^{-1}\left(1 /\left(1-|\lambda|^{2}\right)\right)} \\
& \quad \leq C\left\|C_{\phi}^{g}\right\|_{\mathscr{L} \rightarrow \mathscr{L}^{\varphi}}+2 \frac{\left|\phi^{\prime}(\lambda)\right||g(\lambda)||\phi(\lambda)|}{\varphi^{-1}\left(1 /\left(1-|\lambda|^{2}\right)\right)\left(1-|\phi(\lambda)|^{2}\right)} .
\end{aligned}
$$

Set

$$
h_{a}(z)=\frac{h(\bar{a} z)}{\bar{a}}\left(\log \frac{1}{1-|a|^{2}}\right)^{-1}-\int_{0}^{z} \log \frac{1}{1-\bar{a} w} d w,
$$

for $a \in \mathbb{D}$, such that $|a|>1 / 2$. Then,

$$
\begin{aligned}
& h_{a}^{\prime}(z)=\left(\log \frac{1}{1-\bar{a} z}\right)^{2}\left(\log \frac{1}{1-|a|^{2}}\right)^{-1}-\log \frac{e}{1-\bar{a} z}, \\
& h_{a}^{\prime \prime}(z)=\frac{2 \bar{a}}{1-\bar{a} z}\left(\log \frac{1}{1-\bar{a} z}\right)\left(\log \frac{1}{1-|a|^{2}}\right)^{-1}-\frac{\bar{a}}{1-\bar{a} z} .
\end{aligned}
$$

Similar to the case of $f_{a}$, we have $h_{a} \in \mathscr{Z}$ and $M_{1}=$ $\sup _{1 / 2<|a|<1}\left\|h_{a}\right\|_{\mathscr{L}}<\infty$. From this and by the facts that $h_{a}^{\prime}(a)=0$ and $h_{a}^{\prime \prime}(a)=\bar{a} /\left(1-|a|^{2}\right)$, it follows that

$$
C\left\|C_{\phi}^{g}\right\|_{\mathscr{X} \rightarrow \mathscr{E}^{\varphi}} \geq \frac{\left|\phi^{\prime}(\lambda)\right||g(\lambda)||\phi(\lambda)|}{\varphi^{-1}\left(1 /\left(1-|\lambda|^{2}\right)\right)\left(1-|\phi(\lambda)|^{2}\right)} .
$$

From (71) we have

$$
\begin{aligned}
& \sup _{|\phi(\lambda)|>1 / 2} \frac{\left|\phi^{\prime}(\lambda)\right||g(\lambda)||\phi(\lambda)|}{\varphi^{-1}\left(1 /\left(1-|\lambda|^{2}\right)\right)\left(1-|\phi(\lambda)|^{2}\right)} \\
& <2 \frac{\left|\phi^{\prime}(\lambda)\right||g(\lambda)||\phi(\lambda)|}{\varphi^{-1}\left(1 /\left(1-|\lambda|^{2}\right)\right)\left(1-|\phi(\lambda)|^{2}\right)} \\
& \leq \sup _{|\phi(\lambda)|>1 / 2} C\left\|C_{\phi}^{g}\right\|_{\mathscr{X} \rightarrow \mathscr{I}^{\varphi}}<\infty .
\end{aligned}
$$


By (65) we see that

$$
\begin{gathered}
\sup _{|\phi(\lambda)| \leq 1 / 2} \frac{\left|\phi^{\prime}(\lambda)\right||g(\lambda)|}{\varphi^{-1}\left(1 /\left(1-|\lambda|^{2}\right)\right)\left(1-|\phi(\lambda)|^{2}\right)} \\
\quad \leq \sup _{|\phi(\lambda)| \leq 1 / 2} \frac{4}{3} \frac{\left|\phi^{\prime}(\lambda)\right||g(\lambda)|}{\varphi^{-1}\left(1 /\left(1-|\lambda|^{2}\right)\right)}<\infty .
\end{gathered}
$$

From (72) and (73) we obtain the first inequality of (57). Similarly, from (61) and (68) the second inequality in (57) follows as desired.

Theorem 7. Let $\varphi:[0, \infty) \rightarrow[0, \infty)$ be an $\mathcal{N}$-function, $g \in$ $H(\mathbb{D})$, and $\phi$ an analytic self-map of $\mathbb{D}$. Then $C_{\phi}^{g}: \mathscr{Z} \rightarrow \mathscr{Z}^{\varphi}$ is compact if and only if $C_{\phi}^{g}: \mathscr{Z} \rightarrow \mathscr{Z}^{\varphi}$ is bounded and

$$
\begin{gathered}
\lim _{|\phi(z)| \rightarrow 1} \frac{\left|\phi^{\prime}(z)\right||g(z)|}{\varphi^{-1}\left(1 /\left(1-|z|^{2}\right)\right)\left(1-|\phi(z)|^{2}\right)}=0, \\
\lim _{|\phi(z)| \rightarrow 1} \frac{\left|g^{\prime}(z)\right| \log \left(e /\left(1-|\phi(z)|^{2}\right)\right)}{\varphi^{-1}\left(1 /\left(1-|z|^{2}\right)\right)}=0 .
\end{gathered}
$$

Proof. Suppose that $C_{\phi}^{g}: \mathscr{Z} \rightarrow \mathscr{Z}^{\varphi}$ is compact. It is clear that $C_{\phi}^{g}: \mathscr{Z} \rightarrow \mathscr{Z}$ is bounded. Let $\left\{Z_{n}\right\}_{n \in N}$ be a sequence in $\mathbb{D}$ such that $\left|\phi\left(z_{n}\right)\right| \rightarrow 1$ as $n \rightarrow \infty$ and $\left(f_{n}\right)_{n \in N}$ defined by

$$
\begin{aligned}
f_{n}(z)= & \frac{\phi\left(z_{n}\right) z-1}{\bar{\phi}\left(z_{n}\right)} \\
& \times\left[1+\log \frac{e}{1-\overline{\phi\left(z_{n}\right)} z}\right]\left(\log \frac{e}{1-\left|\phi\left(z_{n}\right)\right|^{2}}\right)^{-1} .
\end{aligned}
$$

Then $\sup _{n \in N}\left\|f_{n}\right\|_{\mathscr{L}}<\infty$, and $f_{n} \rightarrow 0$ uniformly on compact subsets of $\mathbb{D}$ as $n \rightarrow \infty$. Since $C_{\phi}^{g}: \mathscr{Z} \rightarrow \mathscr{Z}^{\varphi}$ is compact, it gives $\lim _{n \rightarrow \infty}\left\|C_{\phi}^{g} f_{n}\right\|_{\mathscr{X}^{\varphi}}=0$. Note that

$$
\begin{gathered}
f_{n}^{\prime}\left(\phi\left(z_{n}\right)\right)=\log \frac{e}{1-\left|\phi\left(z_{n}\right)\right|^{2}}, \\
f_{n}^{\prime \prime}\left(\phi\left(z_{n}\right)\right)=\frac{2 \overline{\phi\left(z_{n}\right)}}{1-\left|\phi\left(z_{n}\right)\right|^{2}} .
\end{gathered}
$$

We have

$$
\begin{aligned}
\left\|C_{\phi}^{g} f_{n}\right\|_{\mathscr{E}^{\varphi}} \geq & \frac{\left|\phi^{\prime}\left(z_{n}\right)\right|\left|g\left(z_{n}\right)\right|\left|\phi\left(z_{n}\right)\right|}{\varphi^{-1}\left(1 /\left(1-\left|z_{n}\right|^{2}\right)\right)\left(1-\left|\phi\left(z_{n}\right)\right|^{2}\right)} \\
& -\frac{\left|g^{\prime}\left(z_{n}\right)\right| \log \left(e /\left(1-\left|\phi\left(z_{n}\right)\right|^{2}\right)\right)}{\varphi^{-1}\left(1 /\left(1-\left|z_{n}\right|^{2}\right)\right)},
\end{aligned}
$$

and, consequently,

$$
\begin{gathered}
\lim _{\left|\phi\left(z_{n}\right)\right| \rightarrow 1} \frac{\left|\phi^{\prime}\left(z_{n}\right)\right|\left|g\left(z_{n}\right)\right|\left|\phi\left(z_{n}\right)\right|}{\varphi^{-1}\left(1 /\left(1-\left|z_{n}\right|^{2}\right)\right)\left(1-\left|\phi\left(z_{n}\right)\right|^{2}\right)} \\
\quad=\lim _{\left|\phi\left(z_{n}\right)\right| \rightarrow 1} \frac{\left|g^{\prime}\left(z_{n}\right)\right| \log \left(e /\left(1-\left|\phi\left(z_{n}\right)\right|^{2}\right)\right)}{\varphi^{-1}\left(1 /\left(1-\left|z_{n}\right|^{2}\right)\right)} .
\end{gathered}
$$

If one of these two limits exists, set

$$
\begin{aligned}
h_{n}(z)= & \frac{h\left(\overline{\phi\left(z_{n}\right)} z\right)}{\phi\left(z_{n}\right)}\left(\log \frac{e}{1-\left|\phi\left(z_{n}\right)\right|^{2}}\right)^{-1} \\
& -\left(\log \frac{e}{1-\left|\phi\left(z_{n}\right)\right|^{2}}\right)^{-2} \int_{0}^{z} \log ^{3} \frac{e}{1-\overline{\phi\left(z_{n}\right)} w} d w
\end{aligned}
$$

then $h_{n}^{\prime}\left(\phi\left(z_{n}\right)\right)=0, \sup _{n \in N}\left\|h_{n}\right\|_{\mathscr{Z}} \leq C$, and $h_{n}$ converges to 0 uniformly on compact subsets of $\mathbb{D}$ as $n \rightarrow \infty$. Since $C_{\phi}^{g}$ : $\mathscr{Z} \rightarrow \mathscr{Z}^{\varphi}$ is compact, we have

$$
\lim _{n \rightarrow \infty}\left\|C_{\phi}^{g} h_{n}\right\|_{\mathscr{Z}^{\varphi}}=0 .
$$

On the other hand,

$$
\frac{\left|\phi^{\prime}\left(z_{n}\right)\right|\left|g\left(z_{n}\right)\right|\left|\phi\left(z_{n}\right)\right|}{\varphi^{-1}\left(1 /\left(1-\left|z_{n}\right|^{2}\right)\right)\left(1-\left|\phi\left(z_{n}\right)\right|^{2}\right)} \leq\left\|C_{\phi}^{g} h_{n}\right\|_{\mathscr{E}^{\varphi}} .
$$

Hence

$$
\lim _{n \rightarrow \infty} \frac{\left|\phi^{\prime}\left(z_{n}\right)\right|\left|g\left(z_{n}\right)\right|\left|\phi\left(z_{n}\right)\right|}{\varphi^{-1}\left(1 /\left(1-\left|z_{n}\right|^{2}\right)\right)\left(1-\left|\phi\left(z_{n}\right)\right|^{2}\right)}=0 .
$$

Therefore

$$
\begin{gathered}
\lim _{\left|\phi\left(z_{n}\right)\right| \rightarrow 1} \frac{\left|\phi^{\prime}\left(z_{n}\right)\right|\left|g\left(z_{n}\right)\right|\left|\phi\left(z_{n}\right)\right|}{\varphi^{-1}\left(1 /\left(1-\left|z_{n}\right|^{2}\right)\right)\left(1-\left|\phi\left(z_{n}\right)\right|^{2}\right)} \\
=\lim _{k \rightarrow \infty} \frac{\left|\phi^{\prime}\left(z_{n}\right)\right|\left|g\left(z_{n}\right)\right|\left|\phi\left(z_{n}\right)\right|}{\varphi^{-1}\left(1 /\left(1-\left|z_{n}\right|^{2}\right)\right)\left(1-\left|\phi\left(z_{n}\right)\right|^{2}\right)}=0 .
\end{gathered}
$$

This together with (78) implies

$$
\lim _{\left|\phi\left(z_{n}\right)\right| \rightarrow 1} \frac{\left|g^{\prime}\left(z_{n}\right)\right| \log \left(e /\left(1-\left|\phi\left(z_{n}\right)\right|^{2}\right)\right)}{\varphi^{-1}\left(1 /\left(1-\left|z_{n}\right|^{2}\right)\right)}=0 .
$$

The implication follows from the last two equalities.

Conversely, assume that $C_{\phi}^{g}: \mathscr{Z} \rightarrow \mathscr{Z}^{\varphi}$ is bounded and (74) holds. From the proof of Theorem 6 we have

$$
\begin{aligned}
& C_{1}=\sup _{z \in \mathbb{D}} \frac{\left|g^{\prime}(z)\right|}{\varphi^{-1}\left(1 /\left(1-|z|^{2}\right)\right)}<\infty, \\
& C_{2}=\sup _{z \in \mathbb{D}} \frac{\left|\phi^{\prime}(z)\right|\left|g^{\prime}(z)\right|}{\varphi^{-1}\left(1 /\left(1-|z|^{2}\right)\right)}<\infty .
\end{aligned}
$$


On the other hand, from (74), we have that, for every $\varepsilon>0$, there is a $\delta \in(0,1)$, such that

$$
\begin{gathered}
\frac{\left|\phi^{\prime}(z)\right||g(z)|}{\varphi^{-1}\left(1 /\left(1-\left|z_{n}\right|^{2}\right)\right)\left(1-\left|\phi\left(z_{n}\right)\right|^{2}\right)}<\varepsilon, \\
\frac{\left|g^{\prime}(z)\right| \log \left(e /\left(1-|\phi(z)|^{2}\right)\right)}{\varphi^{-1}\left(1 /\left(1-\left|z_{n}\right|^{2}\right)\right)}<\varepsilon,
\end{gathered}
$$

when $\delta<|\phi(z)|<1$.

Assume that $\left\{f_{n}\right\}_{n \in N}$ is a sequence in $\mathscr{Z}$ such that $\sup _{n \in N}\left\|f_{n}\right\|_{\mathscr{Z}} \leq 1$ and $f_{n}$ converges to 0 uniformly on compact subsets of $\mathbb{D}$ as $n \rightarrow \infty$. Let $k=\{z \in \mathbb{D}: \mid \phi(z) \leq$ $\delta \mid\}$. Then by (85) and (86) it follows that

$$
\begin{aligned}
& \left\|C_{\phi}^{g} f_{n}\right\|_{\mathscr{E}^{\varphi}} \\
& =\sup _{z \in \mathbb{D}} \frac{\left|\left(C_{\phi}^{g} f_{n}\right)^{\prime \prime}(z)\right|}{\varphi^{-1}\left(1 /\left(1-|z|^{2}\right)\right)} \\
& =\sup _{z \in \mathbb{D}}\left(\frac{\left|f_{n}^{\prime \prime}(\phi(z))\right|\left|\phi^{\prime}(z)\right||g(z)|+\left|f_{n}^{\prime}(\phi(z))\right|\left|g^{\prime}(z)\right|}{\varphi^{-1}\left(1 /\left(1-|z|^{2}\right)\right)}\right) \\
& \leq \sup _{z \in K}\left(\frac{\left|f_{n}^{\prime \prime}(\phi(z))\right|\left|\phi^{\prime}(z)\right||g(z)|+\left|f_{n}^{\prime}(\phi(z))\right|\left|g^{\prime}(z)\right|}{\varphi^{-1}\left(1 /\left(1-|z|^{2}\right)\right)}\right) \\
& +\sup _{z \in \mathbb{D} \backslash K}\left(\frac{\left|f_{n}^{\prime \prime}(\phi(z))\right|\left|\phi^{\prime}(z)\right||g(z)|+\left|f_{n}^{\prime}(\phi(z))\right|\left|g^{\prime}(z)\right|}{\varphi^{-1}\left(1 /\left(1-|z|^{2}\right)\right)}\right) \\
& \leq C_{2} \sup _{z \in K}\left|f_{n}^{\prime \prime}(\phi(z))\right|+C_{1} \sup _{z \in K}\left|f_{n}^{\prime}(\phi(z))\right| \\
& +C \sup _{z \in \mathbb{D} \backslash K \varphi^{-1}\left(1 /\left(1-\left|z_{n}\right|^{2}\right)\right)\left(1-\left|\phi\left(z_{n}\right)\right|^{2}\right)}\left\|f_{n}\right\|_{\mathscr{Z}} \\
& +C \sup _{z \in \mathbb{D} \backslash K} \frac{\left|g^{\prime}(z)\right| \log \left(e /\left(1-|\phi(z)|^{2}\right)\right)}{\varphi^{-1}\left(1 /\left(1-\left|z_{n}\right|^{2}\right)\right)}\left\|f_{n}\right\|_{\mathscr{Z}} \\
& \leq C_{2} \sup _{|w| \leq \delta}\left|f^{\prime \prime}(w)\right|+C_{1} \sup _{|w| \leq \delta}\left|f_{n}^{\prime}(w)\right|+2 C \varepsilon L .
\end{aligned}
$$

So we obtain

$$
\begin{aligned}
\left\|C_{\phi}^{g} f_{n}\right\|_{\mathscr{E}^{\varphi}}= & \left|f_{n}^{\prime}(\phi(0))\right||f(0)|+\sup _{z \in \mathbb{D}} \frac{\left|\left(C_{\phi}^{g} f_{n}\right)^{\prime \prime}(z)\right|}{\left.\varphi^{-1}\left(1-|z|^{2}\right)\right)} \\
\leq & C_{2} \sup _{|w| \leq \delta}\left|f_{n}^{\prime \prime}(w)\right|+C_{1} \sup _{|w| \leq \delta}\left|f_{n}^{\prime}(w)\right|+2 C \varepsilon\left\|f_{n}\right\|_{\mathscr{E}} \\
& +\left|f_{n}^{\prime}(\phi(0))\right||g(0)| .
\end{aligned}
$$

By the Cauchy estimate, if $\left\{f_{n}\right\}_{n \in N}$ is a sequence converging to zero on compact subsets of $\mathbb{D}$, then the sequence $\left\{f_{n}\right\}_{n \in N}$ and $\left\{f_{n}^{\prime \prime}\right\}_{n \in N}$ also converges to zero on compact subsets of $\mathbb{D}$ as $n \rightarrow \infty$. In particular, since $K$ is compact it follows that $\lim _{n \rightarrow \infty} \sup _{w \in K}\left|f_{n}^{\prime}(w)\right|=0$ and $\lim _{n \rightarrow \infty} \sup _{w}\left|f^{\prime \prime}(w)\right|=0$. Using these facts and letting $n \rightarrow \infty$ in the last inequality, we obtain that

$$
\lim _{n \rightarrow \infty}\left\|C_{\phi}^{g}\right\|_{\mathscr{L}^{\varphi}} \leq 2 C \varepsilon L
$$

Since $\varepsilon$ is an arbitrary positive number it follows that the last limit is equal to zero. Employing Lemma 3 the implication follows.

\section{Conflict of Interests}

The authors declare that there is no conflict of interests regarding the publication of this paper.

\section{Acknowledgments}

The authors would like to thank Professor Shengjian Wu for helpful conversations. Part of the work was done during the first author stay at Peking University, in the spring of 2014. The authors also thank the referee for some very valuable suggestions and comments that significantly improved the presentation of the paper. The work is supported in part by CNSF (Grant no. 11101099, Grant no. 11161007, and Grant no. 11171080), Guizhou Foundation for Science and Technology (Grant no. (2012) 2273 and no. (2014) 2044), Guizhou technology foundation for selected overseas scholars, and Ph.D. research foundation of Guizhou Normal University.

\section{References}

[1] J. M. Anderson, J. Clunie, and C. Pommerenke, "On Bloch functions and normal functions," Journal für Die Reine und Angewandte Mathematik, vol. 270, pp. 12-37, 1974.

[2] K. H. Zhu, "Bloch type spaces of analytic functions," The Rocky Mountain Journal of Mathematics, vol. 23, no. 3, pp. 1143-1177, 1993.

[3] K. R. Attele, "Toeplitz and Hankel operators on Bergman one space," Hokkaido Mathematical Journal, vol. 21, no. 2, pp. 279293, 1992.

[4] S. G. Krantz and S. Stević, "On the iterated logarithmic Bloch space on the unit ball," Nonlinear Analysis: Theory, Methods \& Applications, vol. 71, no. 5-6, pp. 1772-1795, 2009.

[5] S. Stević, "On new Bloch-type spaces," Applied Mathematics and Computation, vol. 215, no. 2, pp. 841-849, 2009.

[6] S. Li and S. Stević, "Generalized composition operators on Zygmund spaces and Bloch type spaces," Journal of Mathematical Analysis and Applications, vol. 338, no. 2, pp. 1282-1295, 2008.

[7] S. Li and S. Stević, "On an integral-type operator from $\omega$ Bloch spaces to $\mu$-Zygmund spaces," Applied Mathematics and Computation, vol. 215, no. 12, pp. 4385-4391, 2010.

[8] J. C. R. Fernández, "Composition operators on Bloch-Orlicz type spaces," Applied Mathematics and Computation, vol. 217, no. 7, pp. 3392-3402, 2010.

[9] J. Shapiro, Composition Operators and Classical Function Theory, Springer, New York, NY, USA, 1993. 
[10] X. Zhu, "Integral-type operators from iterated logarithmic Bloch spaces to Zygmund-type spaces," Applied Mathematics and Computation, vol. 215, no. 3, pp. 1170-1175, 2009.

[11] P. Duren, Theory of Hp Spaces, Dover, 2000.

[12] S. Li and S. Stević, "Products of Volterra type operator and composition operator from $H^{\infty}$ and Bloch spaces to Zygmund spaces," Journal of Mathematical Analysis and Applications, vol. 345, no. 1, pp. 40-52, 2008.

[13] S. Li and S. Stević, "Weighted composition operators from Zygmund spaces into Bloch spaces," Applied Mathematics and Computation, vol. 206, no. 2, pp. 825-831, 2008.

[14] M. M. Rao and Z. D. Ren, Theory of Orlicz Spaces, Marcel Dekker, New York, NY, USA, 1991.

[15] S. Ye and Q. Hu, "Weighted composition operators on the Zygmund space," Abstract and Applied Analysis, vol. 2012, Article ID 462482, 18 pages, 2012.

[16] X. Zhu, "Extended Cesaro opperators from H1 to Zygmund type spaces in the unit ball," Journal of Computational Analysis and Applications, vol. 11, no. 2, pp. 356-363, 2009.

[17] C. Cowen Jr. and B. MacCluer, Composition Operators on Spaces of Analytic Functions, vol. 20, CRC Press, New York, NY, USA, 1995.

[18] B. R. Choe, H. Koo, and W. Smith, "Composition operators on small spaces," Integral Equations and Operator Theory, vol. 56, no. 3, pp. 357-380, 2006.

[19] S. Li and S. Stević, "Volterra-type operators on Zygmund spaces," Journal of Inequalities and Applications, vol. 2007, Article ID 32124, 10 pages, 2007.

[20] S. Stević, "On an integral operator on the unit ball in $\mathbb{C}^{n}$," Journal of Inequalities and Applications, vol. 2005, no. 1, pp. 81-88, 2005.

[21] J. Xiao, "Riemann-stieltjes operators on weighted bloch and bergman spaces of the unit ball," Journal of the London Mathematical Society, vol. 70, no. 1, pp. 199-214, 2004.

[22] R. Yoneda, "The composition operators on weighted Bloch space," Archiv der Mathematik, vol. 78, no. 4, pp. 310-317, 2002. 


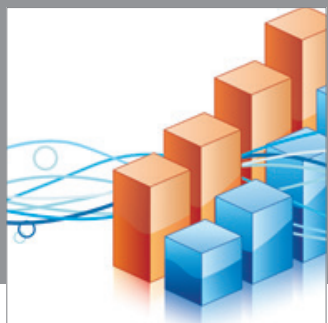

Advances in

Operations Research

mansans

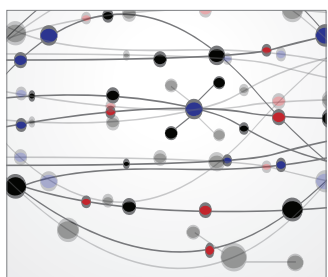

The Scientific World Journal
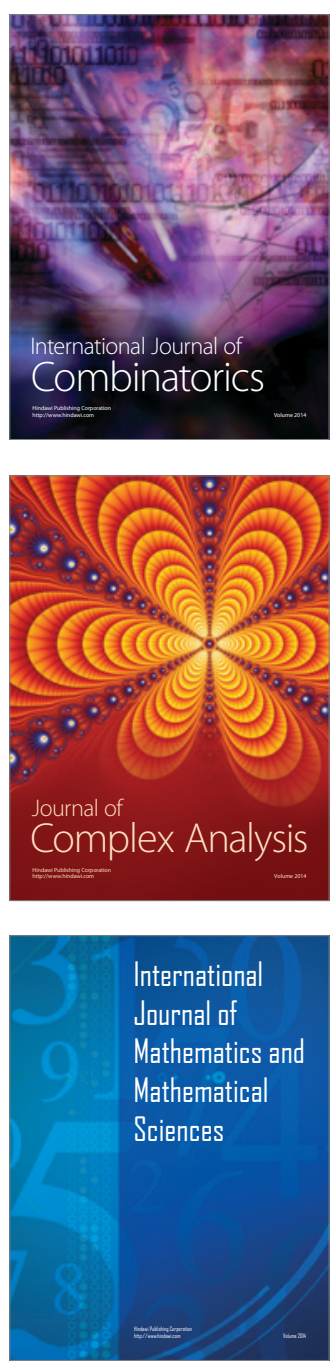
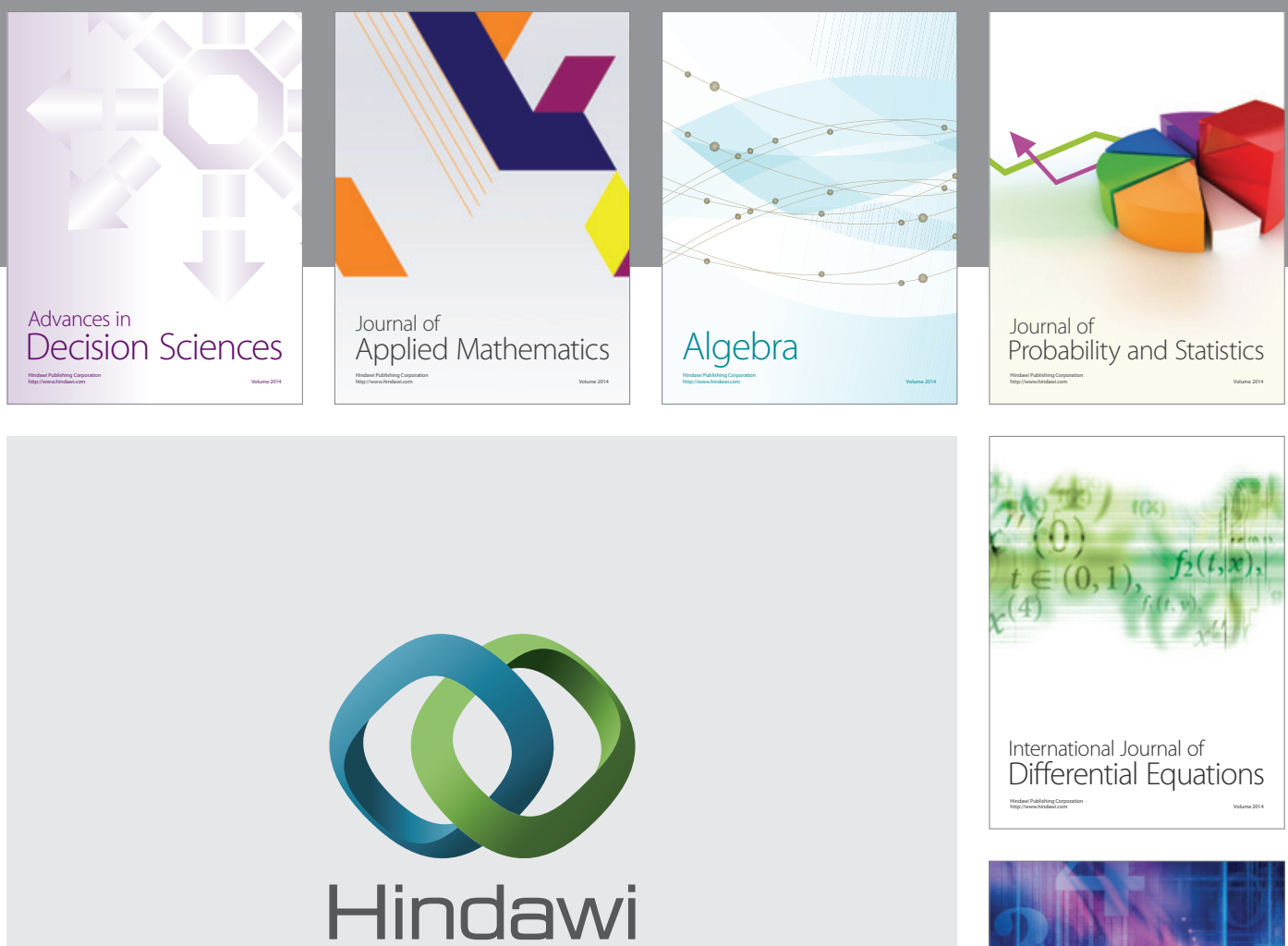

Submit your manuscripts at http://www.hindawi.com
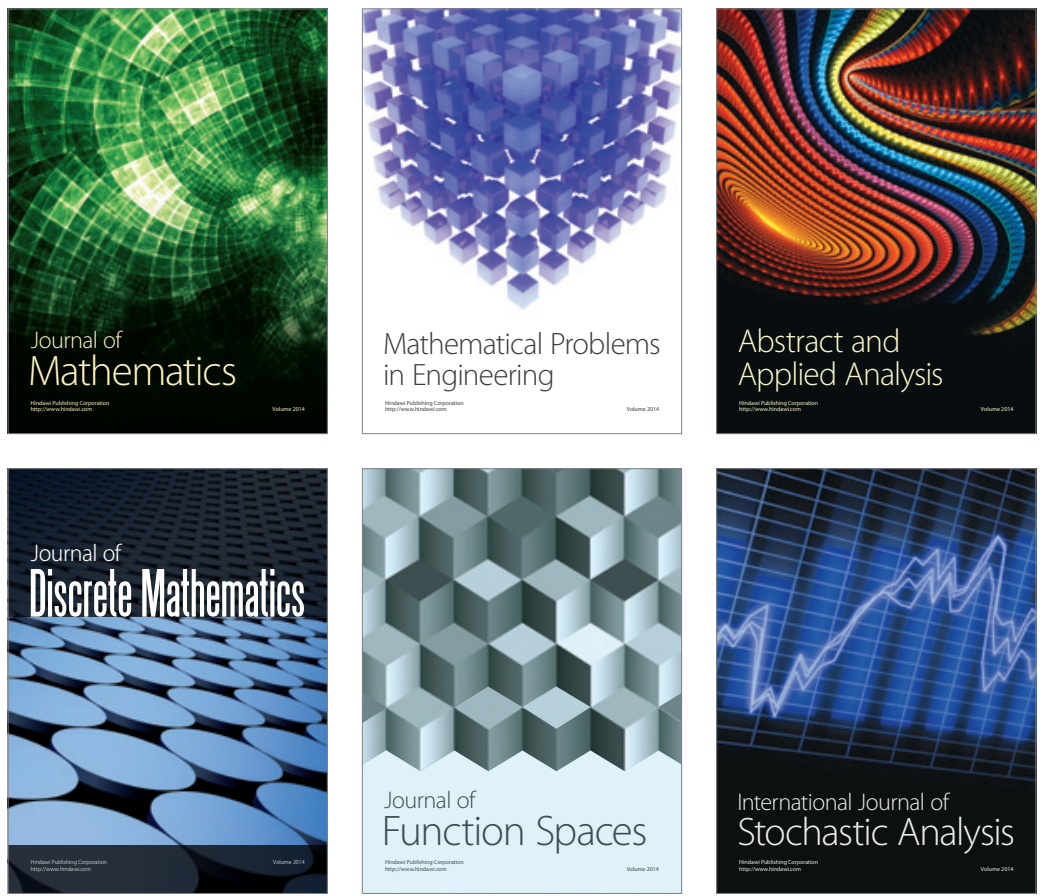

Journal of

Function Spaces

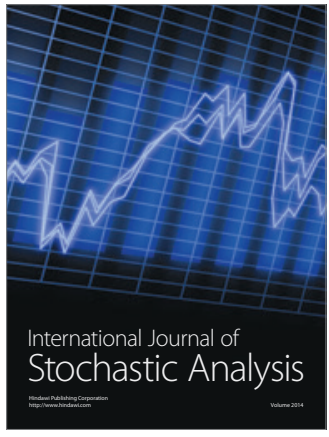

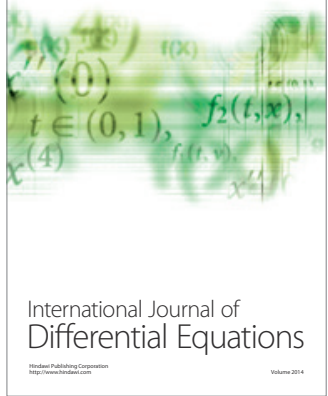
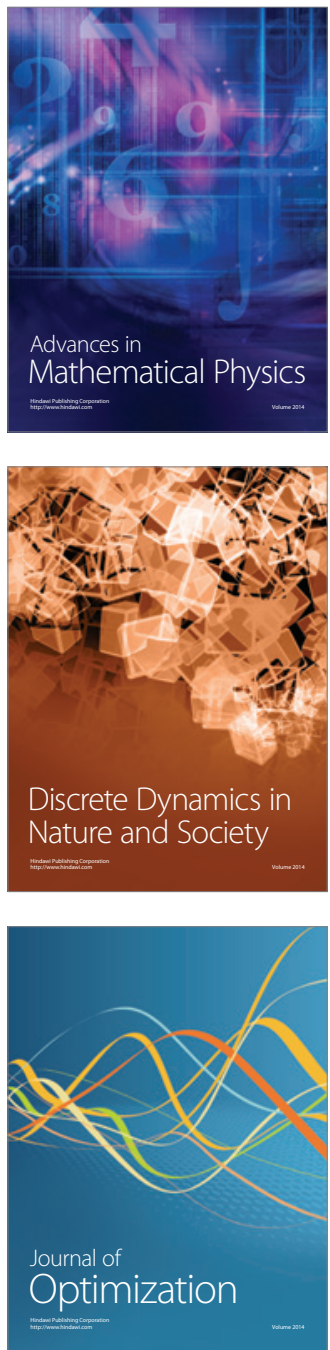